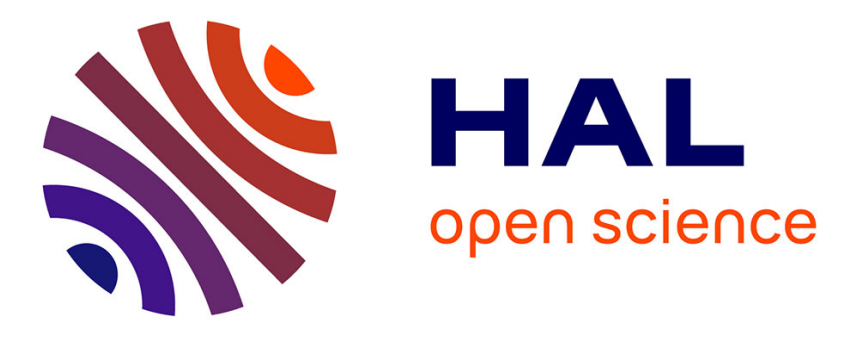

\title{
Manufacturing Cell Simulation Environment for Automated Visual Inspection Using Robot First Report: Fundamental System
}

\author{
Hironori Hibino, Toshihiro Inukai, Yukishige Yoshida
}

\section{To cite this version:}

Hironori Hibino, Toshihiro Inukai, Yukishige Yoshida. Manufacturing Cell Simulation Environment for Automated Visual Inspection Using Robot First Report: Fundamental System. International Conference on Advances in Production Management Systems (APMS), Sep 2011, Stavanger, Norway. pp.171-180, 10.1007/978-3-642-33980-6_21 . hal-01524203

\section{HAL Id: hal-01524203 \\ https://hal.inria.fr/hal-01524203}

Submitted on 17 May 2017

HAL is a multi-disciplinary open access archive for the deposit and dissemination of scientific research documents, whether they are published or not. The documents may come from teaching and research institutions in France or abroad, or from public or private research centers.
L'archive ouverte pluridisciplinaire HAL, est destinée au dépôt et à la diffusion de documents scientifiques de niveau recherche, publiés ou non, émanant des établissements d'enseignement et de recherche français ou étrangers, des laboratoires publics ou privés. 


\title{
Manufacturing Cell Simulation Environment for Automated Visual Inspection Using Robot First Report: Fundamental System
}

\author{
Hironori Hibino $^{1,2,}$ Toshihiro Inukai ${ }^{3}$, Yukishige Yoshida $^{3}$ \\ ${ }^{1}$ Technical Research Institute of JSPMI(Japan Society for the Promotion of Machine \\ Industry), 1-1-12 Hachiman-cho, Higashikurume, Tokyo, Japan, \\ hibino@tri.jspmi.or.jp \\ ${ }^{2}$ Tokyo University of Agriculture and Technology, Tokyo, Japan, \\ ${ }^{3}$ DENSO Wave Inc., Aichi, Japan
}

\begin{abstract}
Recently the industries provide visual inspection processes in the plants for keeping and guaranteeing product quality. Many visual inspection processes are normally operated by the manual visual inspection. The results of the manual visual inspection are often unstable because the results are depended on the inspection worker skill. Currently the automated visual inspection technologies are getting more important to stably keep and guarantee product quality. Specially, the automated visual inspection technologies using robots attract the industries. The robot usually has several industrial cameras and LED lights on its hand. However based on our analysis for the typical implementation procedure of the automated visual inspection technologies using robots, the period to implement the inspection processes are usually very long. The reasons are that there are many adjustment activities in the real plant concurrently concerning the imaging conditions, the robot motion conditions, and the visual inspection conditions. In order to reduce the period to implement the automated visual inspection processes, it is very important to reduce the adjustment activates in the real plant. Therefore it is necessary to develop the simulation technologies to support the adjustment activates on the virtual beforehand. We focus on the manufacturing cell simulation environment for the automated visual inspection using robots. The manufacturing cell simulation environment which provides to support the above adjustments on the virtual even if the robot and the target product are not existed, is proposed and developed. In this paper, the manufacturing cell simulation environment to solve the problems is proposed. Seven requirements for the simulation environment are defined. The fundamental system with five functions to implement the simulation environment is proposed and implemented. Hypothetic fundamental case studies are carried out to confirm effective of our proposed manufacturing cell simulation environment.
\end{abstract}

Keywords: Automated visual inspection, robot, virtual camera, Manufacturing system, simulation.

\section{$1 \quad$ Introduction}

Recently Japanese industries are becoming more important to keep and guarantee product quality even if low skilled workers make the product because the highly skilled workers in Japan are decreasing in the workshop by increasing the highly skilled workers who are close to the retirement age, and the workers who need more education and skill to product are increasing in the globally localization plants [1]. In 
order to solve the problem, the industries provide visual inspection processes in the plants. Many visual inspection processes are normally operated by the manual visual inspection. The results of the manual visual inspection are often unstable because the results are depended on the inspection worker skill. Currently the automated visual inspection technologies are getting more important to stably keep and guarantee product quality. Specially, the automated visual inspection technologies using robots attract the industries. The robot usually has several industrial cameras and LED lights on its hand. The robot precisely inspects the target product while inspecting many inspection points by moving the cameras and lights. The inspection process using the robot is usually implemented as one type of the manufacturing cells. Figure 1 shows the typical automated visual inspection technologies using robots.

However based on our analysis for the typical implementation procedure of the automated visual inspection technologies using robots, the period to implement the inspection processes are usually very long. The reasons are that there are many adjustment activities in the real plant concurrently concerning the imaging conditions, the robot motion conditions, and the visual inspection conditions as follows. Firstly there are many inspection items and points for each product in an inspection process, although there are many kinds of products to inspect in the inspection process. Secondly there are many numbers of adjustments for taking images by the industrial cameras while deciding the efficient robot motions. In order to accurately take the images, it is very important to adjust the focal length for the industrial cameras, to adjust suitable timing to take the images, and to adjust suitable lighting conditions. Thirdly there are many numbers of adjustments for making and evaluating the visual inspection programs while taking the images. Fourthly in the cases to additionally inspect new products, the above adjustments are needed to consider on the workshop while the inspection processes are interrupted during the adjustment activities period.

In order to reduce the period to implement the automated visual inspection processes, it is very important to reduce the adjustment activates in the real plant. Therefore it is necessary to develop the simulation technologies to support the adjustment activates on the virtual beforehand [2][3][4][5][6][7][8]. We focus on the manufacturing cell simulation environment for the automated visual inspection using robots. The manufacturing cell simulation environment which provides to support the above adjustments on the virtual even if the robot and the target product are not existed, is proposed and developed. In this paper, the manufacturing cell simulation environment to solve the problems is proposed. Seven requirements for the simulation environment are defined. The fundamental system with five functions to implement the simulation environment is proposed and implemented. Hypothetic fundamental case studies are carried out to confirm effective of our proposed manufacturing cell simulation environment. 


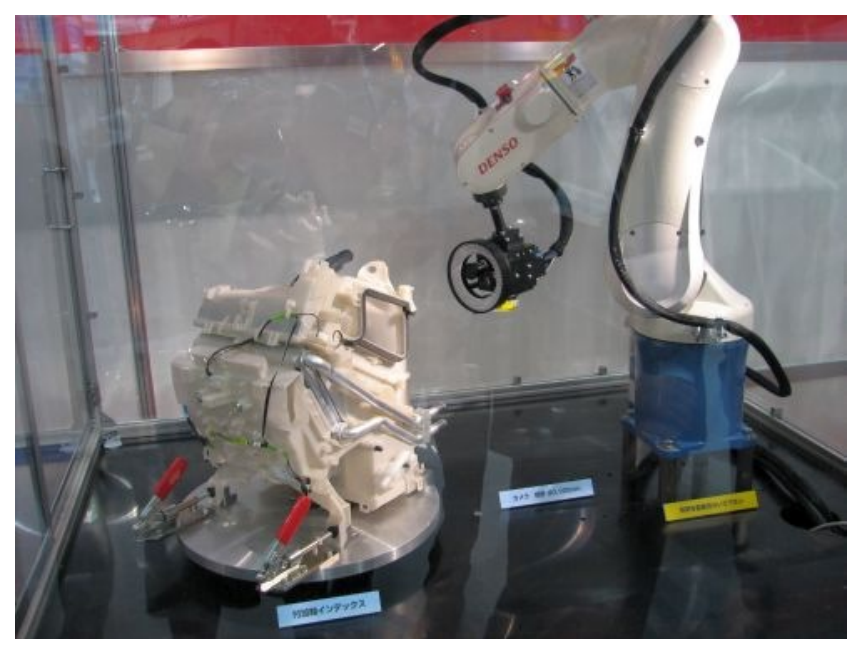

Fig. 1. This figure shows the typical automated visual inspection technologies using robots.

\section{Our Proposed Simulation for Automated Visual Inspection Using Robot}

Based on our analysis for the typical engineering procedure to construct the automated visual inspection using robot, the current engineering procedure to construct the automated visual inspection using robot can be shown as follows. Figure 2 shows the current engineering procedure.

1. The use company engineer gets the target products and defines the inspection items.

2. The engineer for the automated visual inspection makes the visual inspection specification on the desk.

3. The engineer for robot makes the robot program specification and the parts of motion program on the desk.

4. The engineer for robot makes robot motion program in the real plant.

5. The engineer for robot and the engineer for the automated visual inspection are working together and defining the imaging conditions in the real plant by considering the focal length for the industrial cameras, suitable timing to take the images, and suitable lighting conditions while deciding the efficient robot motions.

6. The engineer for the automated visual inspection makes the visual inspection program in the real plant while taking the images.

$80 \%$ of the total lead-time to construct the automated visual inspection is time for the adjustment activities as No.4, 5, and 6 in the real plant repeatedly and concurrently. In the cases to additionally inspect new products, the above adjustments are needed to consider on the real plant while the inspection processes are interrupted during the adjustment activities period.

In order to reduce the period to construct the automated visual inspection using robot, it is very important to reduce the period for the adjustment activates in the real 
plant. Our research focuses on the simulation technologies to solve the problems. We develop the simulation technologies for the automated visual inspection using robots, which support the adjustment activities on the virtual concerning the imaging condition adjustment, the robot motion condition adjustment, and the visual inspection condition adjustment even if the robot and the target product do not exist. Figure 2 shows our proposed engineering procedure using the simulation technologies. The final goal for the manufacturing cell simulation environment is to reduce $80 \%$ of the adjustment activities in the real plant.

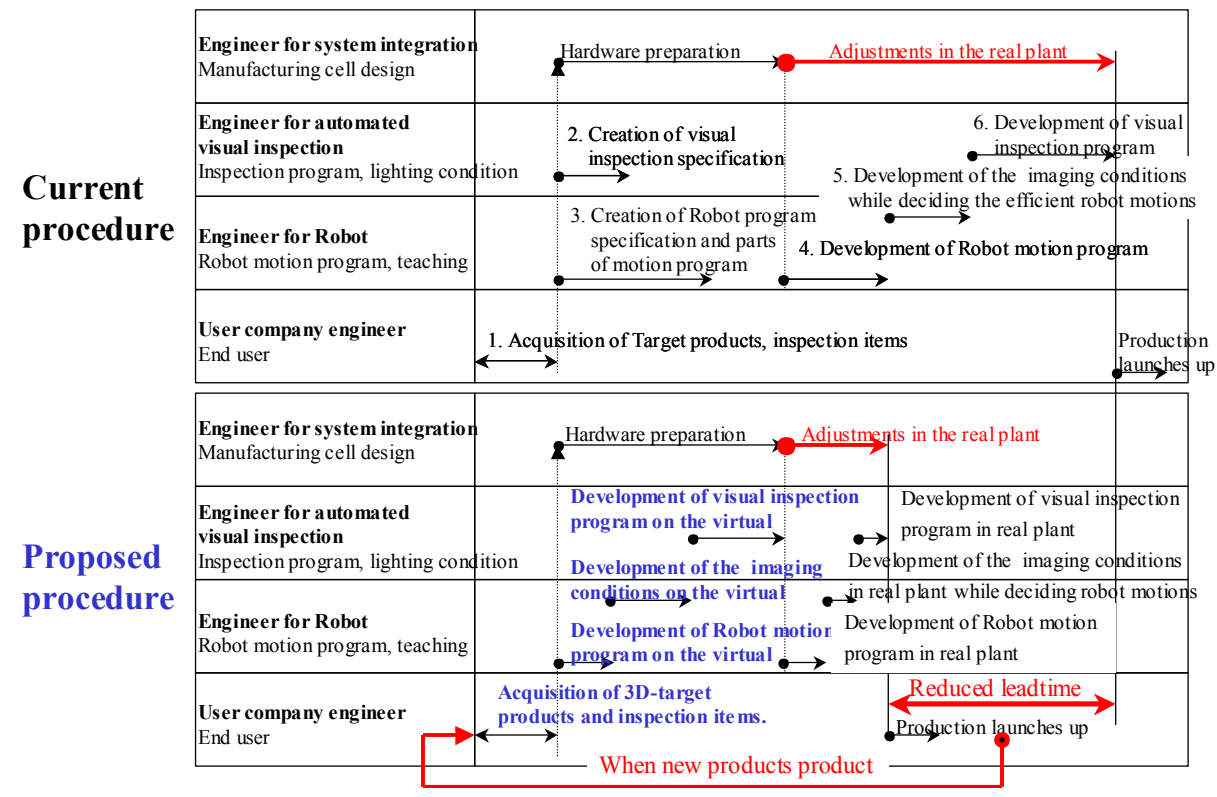

Fig. 2. Engineering procedure to construct automated visual inspection using robot.

\section{Requirements for Manufacturing Cell Simulation Environment for Automated Visual Inspection Using Robot}

On the situations that the robot and the target product do not exist, our proposed simulation supports firstly deciding conditions for taking images on the virtual while arranging the industrial cameras, the lightings and so on, secondly developing the robot motions programs and the control programs for programmable logic controller (PLC), and thirdly developing the visual inspection programs. Figure 3 shows an outline of our proposed simulation. In order to realize the simulation, the necessary requirements are defined as follows. 


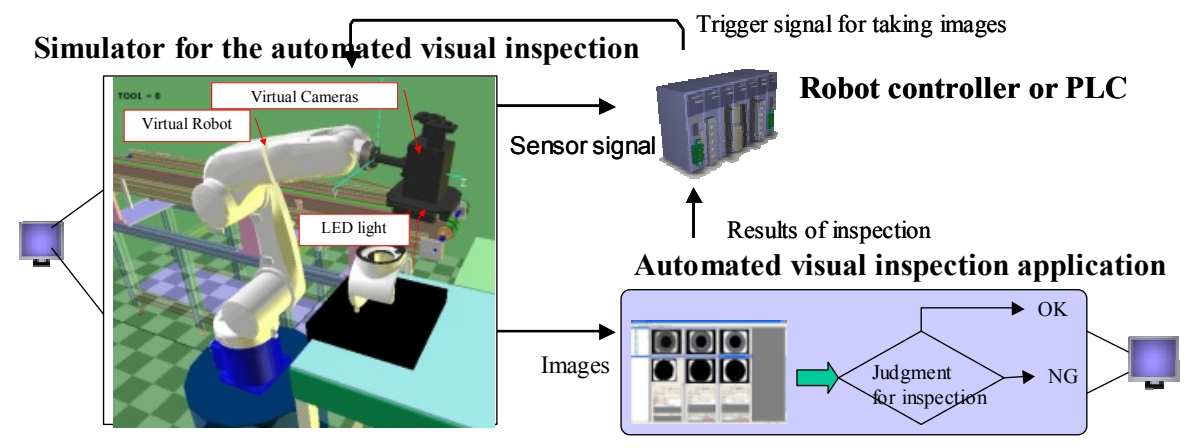

Fig. 3. An outline of our proposed simulation

For deciding conditions for taking images on the virtual while arranging the industrial cameras, the lightings and so on, two requirements are proposed.

1. Inspection condition design support to design suitable inspection conditions for taking images on the virtual while arranging the industrial cameras, the lightings, the robots, and the product.

2. Inspection point design support to design suitable inspection points on the virtual while considering the industrial cameras condition such as the focal length and the wide angle, the robot motion conditions such as the reachable space, and the inspection specifications such as the inspection items.

For developing the robot motions programs and the control programs for programmable logic controller (PLC), three requirements are proposed.

3. Image creation support to take images on the virtual which include grayscale image information by the influence of the lighting conditions, and include the images with the inspection target field and outside of the inspection target field such as background by considering the focal length and the wide angle.

4. Device control program creation support to make automated control programs for devices such as ladder programs for PLC.

5. Robot control program creation support to make robot control programs while considering suitable robot trajectories without interferences and with high productivity.

For developing the visual inspection programs, two requirements are proposed.

6. Visual inspection program creation support to make visual inspection programs using the images by the image creation support.

7. Visual inspection program improvement support to improve visual inspection programs while using failure images which are taken on the virtual such as images with scratches, lack of holes, and so on. 


\section{Fundamental System for Manufacturing Cell Simulation Environment for Automated Visual Inspection Using Robot}

In order to realize seven requirements for the simulation environment, the fundamental system with five functions is proposed. Five functions are defined as follows.

1. Manufacturing cell simulation function (Visual inspection imaging function) to take images with an inspection target field by virtual industrial cameras on a virtual robot in the computer graphic space while synchronizing the virtual robot motion. Then the virtual camera images a virtual product model created by threedimensional CAD.

2. Image transfer function to transfer the created image to visual inspection application.

3. Real time synchronization function to synchronize among the manufacturing cell simulation and real controller equipment such as PLC.

4. Distributed simulation function to synchronize among the manufacturing cell simulation and the robot simulation.

5. Failure image creation function to make necessary kinds of failure images using a virtual product model created by three-dimensional CAD such as images with scratches, lack of holes, and so on.

In order to implement the manufacturing cell simulation function, the manufacturing cell simulator (EMU) is developed. EMU mainly provides six functions.

In order to implement the image transfer function, the soft-wiring system is developed. The soft-wiring system provides to wire image information such as bitmap information between EMU and the visual inspection applications with real time. The semi-standard industrial middleware ORIN [9] is used for the soft wiring system.

In order to implement the real time synchronization function, the soft-wiring system is also developed. The soft-wiring system provides to wire control information such as I/O, BCD parameter between EMU and real controller equipment such as PLC with real time.

In order to implement the distributed simulation function, the conservative type of synchronization mechanisms [10] is used and implemented.

In order to implement the failure image creation function, the failure image creation methods are proposed and implemented on EMU.

Figure 4 shows an outline of the proposed fundamental system with five functions. Figure 5 shows the system structure of the proposed fundamental system. 


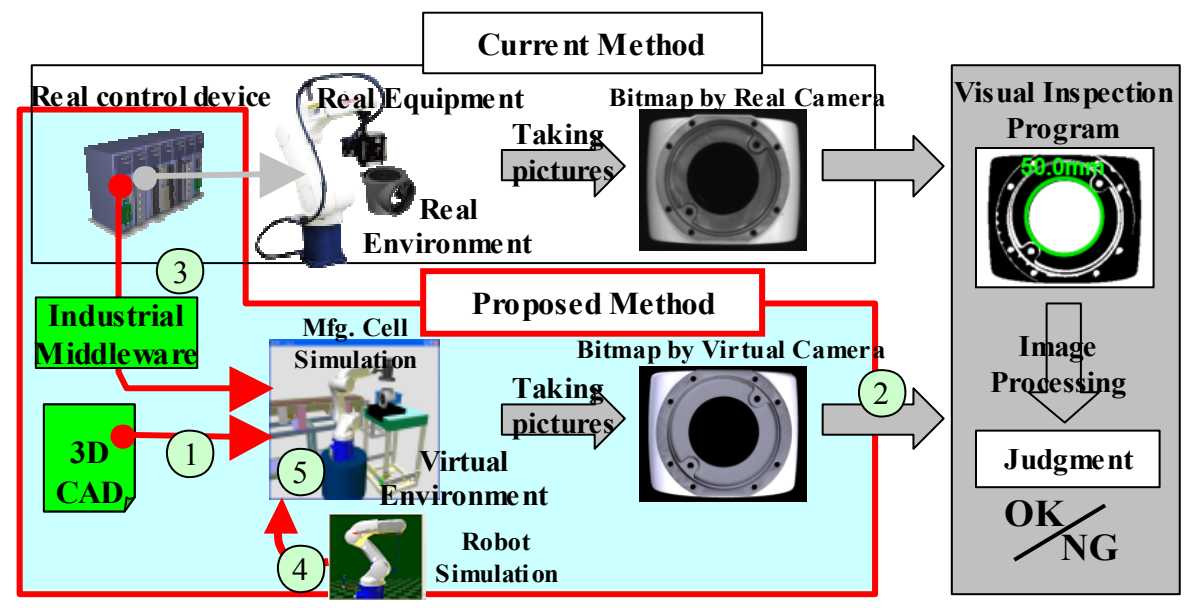

Fig. 4. This figure shows an outline of the proposed fundamental system with five functions.

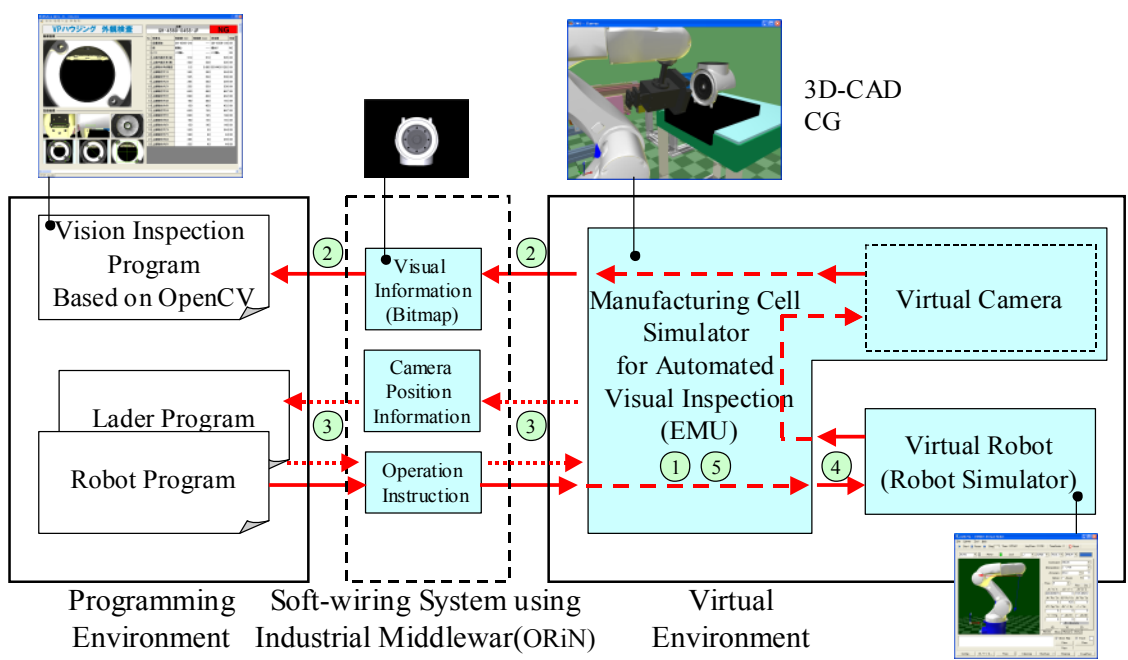

Fig. 5. This figure shows the system structure of the proposed fundamental system.

\section{A Case Study}

A case study was carried out using a manufacturing cell which consists of a robot, three industrial cameras, three LED lights, a visual inspection application. The inspection target product is an aluminum housing which is one of the parts for the industrial robots. The main inspection items are six which are the inspection for product numbers on the housing, scratches, burrs, dimensions, arrangements of things such as holes, layouts, lack of things such as holes. The real aluminum housing and the virtual aluminum housing which is three-dimensional model with 65481 polygons, are provided.

Through this case study, it was confirmed that our proposed fundamental system could be used to support implementation procedure on the virtual even if the robot and the target product are not existed. The implementation procedure on the virtual includes deciding conditions for taking images, deciding the robot motions and to develop control programs for the robot, and developing the visual inspection programs. 
Figure 6 shows our developed EMU. Figure 7 shows the created images by EMU and the real industrial camera for the same inspection point. Figure 8 shows the GUI of the visual inspection program.

The future work is to be continued confirming our proposed fundamental system through other cases.

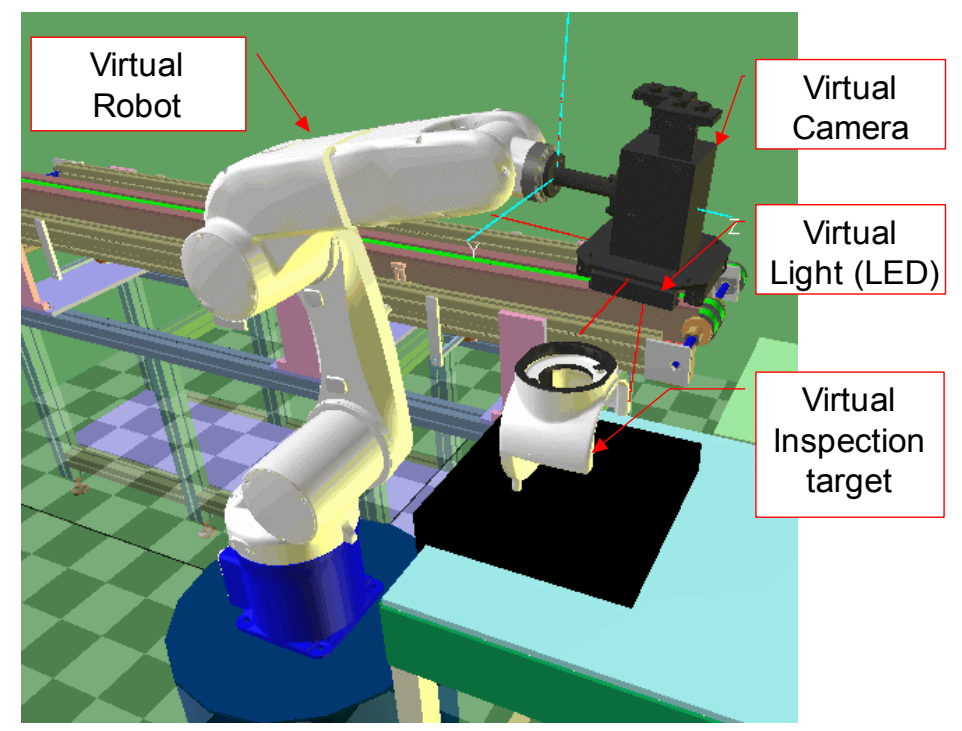

Fig. 6. This figure shows our developed EMU.
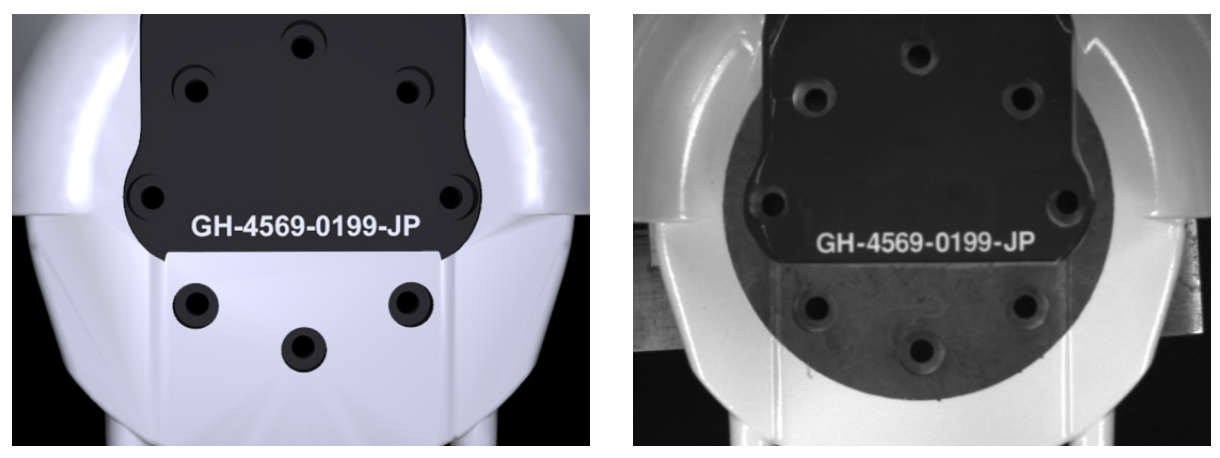

Fig. 7. This figure shows the created images by EMU and the real industrial camera for the same inspection point. 


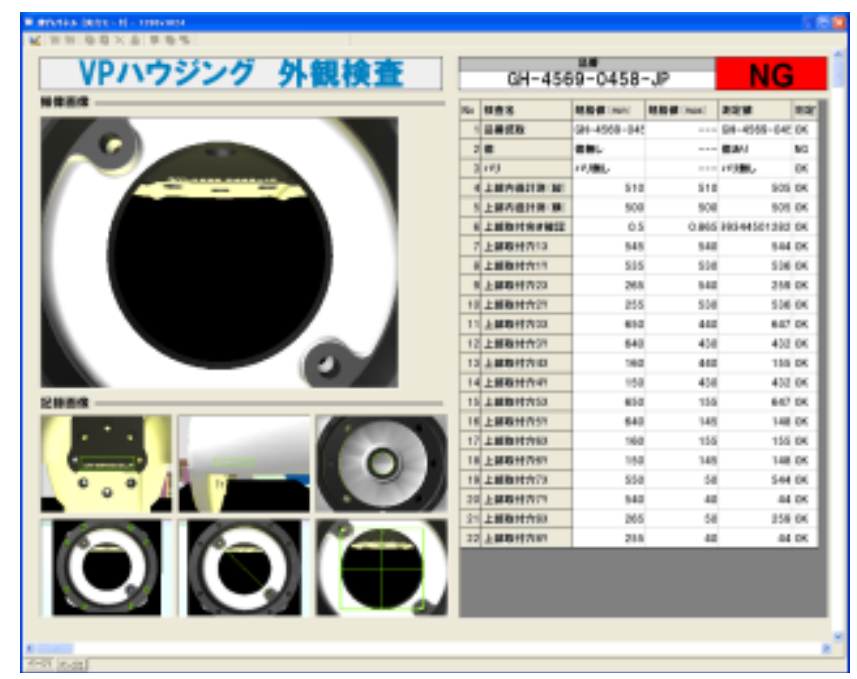

Fig. 8. This figure show the GUI of the visual inspection program.

\section{Conclusion}

In this paper, the manufacturing cell simulation environment for the automated visual inspection using robot is proposed and developed. The manufacturing cell simulation environment provides to support the engineering procedure to implement the automated visual inspection using robot on the virtual even if the robot and the target product are not existed.

The results were:

1. To summarize problems to implement the automated visual inspection using robot.

2. To propose the manufacturing cell simulation environment for the automated visual inspection using robot.

3. To define and clarify necessary seven requirements for the manufacturing cell simulation environment for the automated visual inspection using robot.

4. To define and propose the fundamental system with five functions to implement the simulation environment.

5. To confirm through a case study that the proposed fundamental system could be used to support implementation procedure on the virtual even if the robot and the target product are not existed.

\section{References}

1. Tanaka, K., Hibino, H., Fukuda, Y.: Module Structured Production System. In: 41st CIRP Conference on Manufacturing Systems, pp. 303-308. Springer, Heidelberg, 2008.

2. Hibino, H., Y. Fukuda, Y.: Emulation in Manufacturing Engineering Processes. In: 2008 Winter Simulation Conference, pp. 1785-1793. ISBN:978-1-4244-2708-6, 2008. 
3. Hibino, H., Fukuda, Y., Fujii, S., Kojima, F., Mitsuyuki, K., Yura, Y.: The Development of an Object-oriented Simulation System based on the Thought Process of the Manufacturing System Design. I. J. Production Economics, 60, 343-351, 1999.

4. Hibino, H., Fukuda, Y.: A User Support System for Manufacturing System Design Using Distributed Simulation. Production Planning and Control, 17, 128-142, 2006.

5. Hibino, H., Fukuda, Y., Yura, Y., Mitsuyuki, K., Kaneda, K.: Manufacturing Adapter of Distributed Simulation Systems Using HLA. In: 2002 Winter Simulation Conference, pp. 1099-1109. Institute of Electrical and Electronics Engineers, Inc.. New Jersey, 2002.

6. Hibino, H., Fukuda, Y.: A Synchronization Mechanism without Rollback Function for Distributed Manufacturing Simulation Systems, J. Japan Society of Mechanical Engineers, 68, 2472-2478 [in Japanese], 2002.

7. Hibino, H., Inukai, T., Fukuda, Y.: Efficient Manufacturing System Implementation based on Combination between Real and Virtual Factory. I. J. Production Research, 44, 3897-3915, 2006.

8. Hibino, H.: Simulation Environment for Efficient Manufacturing System Design and Implementation Using Network Middleware ORiN and HLA. J. Society of Instrument and Control Engineers, 46, 560-545 [in Japanese], 2007.

9. ORiN, http://www.orin.jp/, 2011.

10. Kuhl, F., Weatherly, R., Dahmann, J.: Creating Computer Simulation Systems, Prentice Hall, ISBN 0-13-022511-8, 2000. 